\title{
Pressing Control Design Based on Analysis of Metal Temperature and Flow in Sand Mold Press Casting
}

\author{
Ryosuke Tasaki ${ }^{1, a^{*}}$, Hideto Seno ${ }^{1}$, \\ Kunihiro Hashimoto ${ }^{2}$ and Kazuhiko Terashima ${ }^{1}$
}

\author{
${ }^{1}$ Toyohashi University of Technology, 1-1 Hibarigaoka, Tempaku, Toyohashi, Aichi, Japan \\ ${ }^{2}$ Sintokogio, Ltd., 3-1, Honohara, Toyokawa, Aichi, Japan \\ atasaki@me.tut.ac.jp
}

Keywords: Press casting, Filling control, Quality improvement, Iron, Sand mold

\begin{abstract}
The sand mold press casting is a novel iron casting process, which developed by our group in recent years. The proposed method can cast iron into complex shapes with a high yield over $90 \%$ and produces high-quality products by filling control of molten metal during pressing motion. However, molten metal inside mold is cooled down by heat transfer to the molds and atmosphere, and often causes solidification before the end of press. Then, the pressing movement of the upper mold is blocked by the solidified metal. Therefore, to avoid the solidification during pressing sand mold, metal temperature must be heated properly to estimate the filling temperature decrease behavior based on analysis results of CFD simulator, FLOW-3D. The necessary condition not to solidify molten metal before the end of press has been found. It was made clear that the mold must be heated up to the necessary temperature beforehand in the pressing process. Furthermore, optimum velocity reference with specified pressure constraints has been designed to prevent casting defect such as penetration and also to minimize the temperature decrease. In this paper, optimum velocity control of servo cylinder considering the both of allowable pressure of molten metal and starting time of solidification is proposed by using a theoretical approach of Model Predictive Control: MPC method. The effectiveness of the proposed control system has been demonstrated by computer simulation and experiments using a laboratory scale machine with molten metal of casting iron.
\end{abstract}

\section{Introduction}

The sand mold press casting method has been developed by our group in recent years. In this process, a casting mold made of sand is split into upper and lower parts, and molten metal is poured into a lower mold by a ladle first, then the upper mold is lowered toward the lower mold, and finally the two molds are matched. These are the benefits of a counter-gravity casting capable of yielding defect-free metal casting [1]. The main advantage of the press casting compared with the similar casting methods is its potential of high-speed filling process considering metal pressure and flow controls without proving an additional equipment to fill metal to the product cavity. It would be possible to design the short time filling processes with the pouring in a few seconds with the ladle and the pressing within 1 second during mold matching motion as shown in Fig. 1.

Casting defects such as physical metal penetration are often caused by high pressure in a high-speed pressing process. Therefore, pressing motion control is important for defect-free production. As a previous research, the adaptive output control method of a hydraulic cylinder using a direct output force measurement by a load cell was proposed [2]. Also, the robust pressure control system for a control cylinder using by sliding mode control has been proposed [3]. As the research of the press casting, the filling pressure analysis by CFD was investigated and a mathematical model of the pressing pressure was constructed [4, 5]. Furthermore, the pressing control motion has been designed by the predictive control algorithm which predicts a future behavior of pressure before pressing [6]. Here, the main work in the paper is new construction of the filling pressure control system based on fluid simulation analysis of metal temperature and flow in sand mold press casting. 


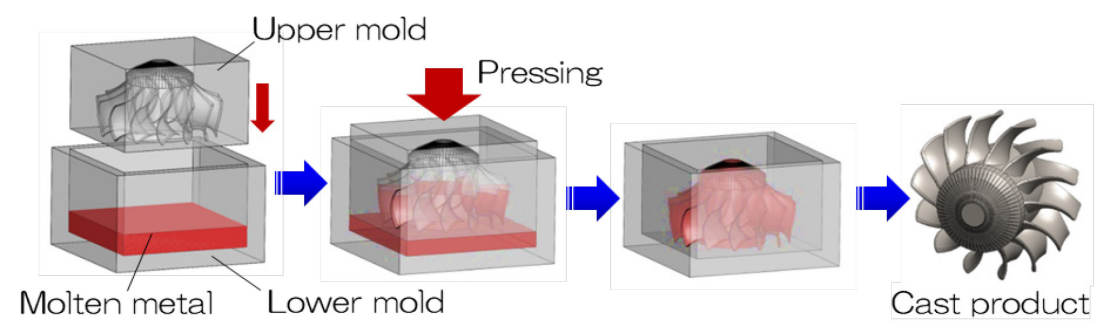

Fig. 1: Filling process in sand mold press casting

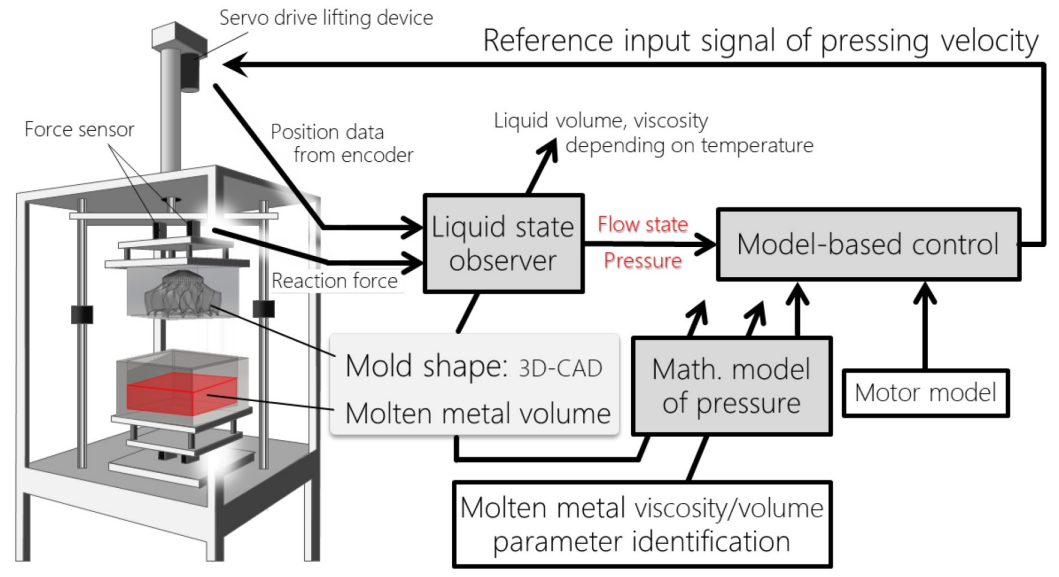

Fig. 2: Filling pressure control system

Molten metal inside molds is cooled down by heat transferring to the molds and atmosphere, and often causes solidification before the end of press. Then, the pressing movement of the upper mold is blocked by the solidified metal. And also, when the pressing velocity of the upper mold is higher, upward flow causes the fountain inside mold. The molten metal fountains from a narrow flow path to the wide path in the cavity, so that flow disturbance and air entrainment are occurred. The filling pressure control system as shown in Fig. 2, is important to properly design the pressing velocity by considering the metal temperature drop estimation and the fountain flow suppression, and to control the filling behaviors of molten metal in real time. Hence, we present a new analysis of the temperature drop for preventing solidification and the upward steady flow of molten metal inside casting cavity, and finally new control design of pressing movement considering the metal temperature drop related to mold's preheating and keeping ideal upward flow without fountain.

\section{Metal Temperature Analysis inside Mold}

To prevent the solidification while pressing sand mold, the metal temperature distributions in the pressing process are analyzed by using CFD simulator. The target metal is cast iron, the liquidus temperature is $1156{ }^{\circ} \mathrm{C}$, the initial temperature of the metal is $1400{ }^{\circ} \mathrm{C}$ and the mold material is quartz sand. In the analysis by CFD, the mesh size is $2 \mathrm{~mm}$ for one piece; the total mesh number is 125000 pieces. The temperature analysis results were compared with preheating temperature of the mold as 20, 200 and $400{ }^{\circ} \mathrm{C}$.

Fig. 3 shows an example of the simulation analysis results of metal flow and temperature distribution. We observe temperature drop on measurement points in the Fig. 4, decide that molten metal inside mold has been solidified in that case of molten metal temperature is lower than the liquidus temperature before end of pressing motion. In the case of the preheated mold temperature is $20^{\circ} \mathrm{C}$, the metal temperature drops under the liquidus line before the end of the press, but in the case of 200 and $400{ }^{\circ} \mathrm{C}$, the metal temperature drops slowly and is still upper than the liquidus line. 

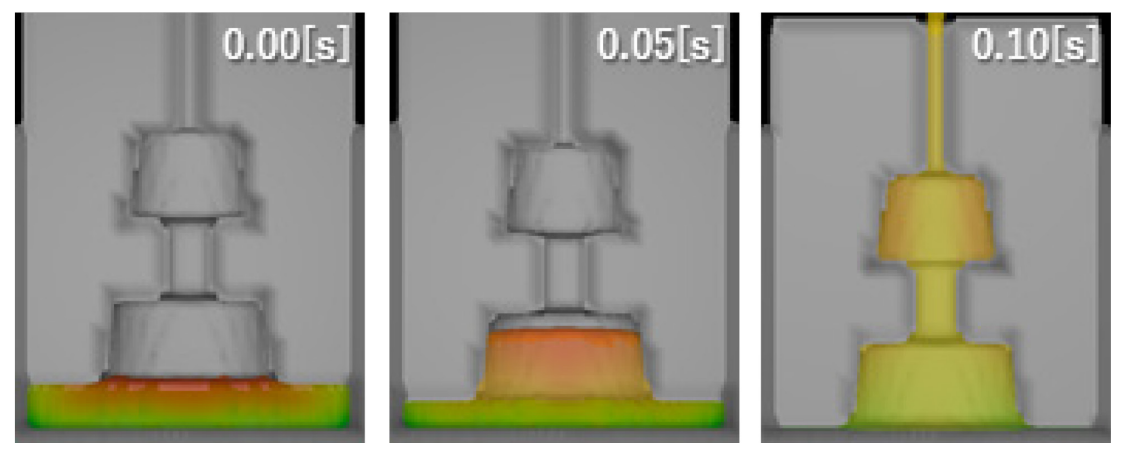

Fig. 3: Analysis of upward flow and temperature distribution

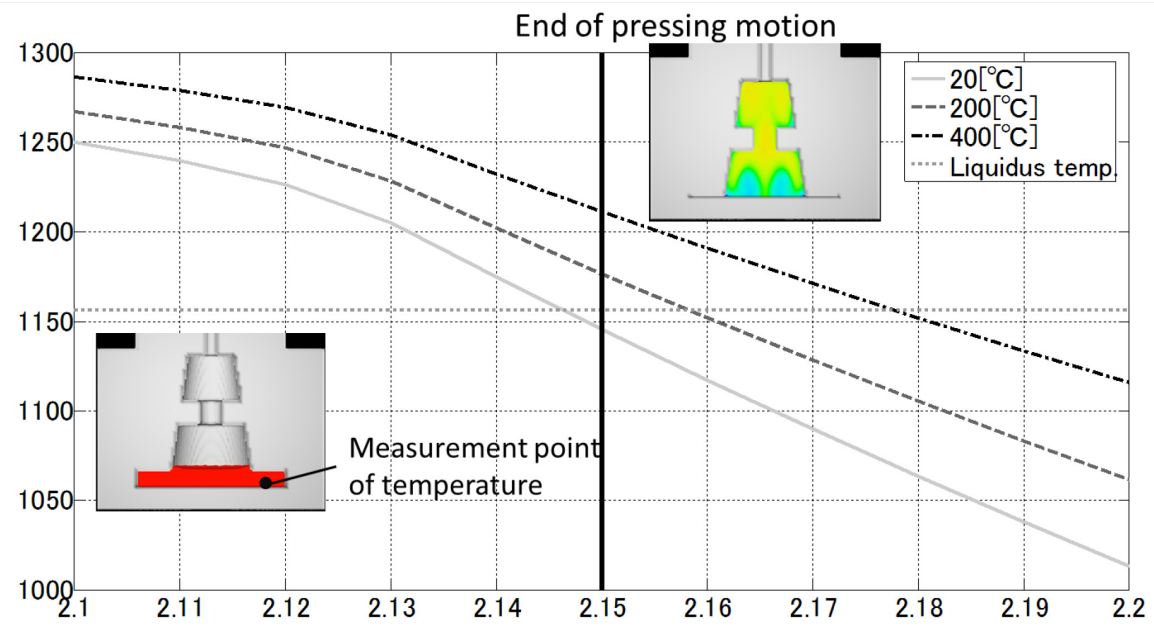

Fig. 4: Time behavior of temperature drop

The press casting experiments was carried out by using molten metal based on the results of the previous temperature analysis. The metal material is cast iron, the initial temperature of the metal is $1400{ }^{\circ} \mathrm{C}$. We compared two conditions that the preheated mold temperature is $200{ }^{\circ} \mathrm{C}$ and without preheating. Table 1 shows the measurement result of the burr thickness at bottom of the product. In the case of the preheated mold temperature, the burr thickness becomes thin clearly.

Table 1: Burr thickness at bottom of cast product

\begin{tabular}{|c|c|}
\hline Without preheating & $1.2[\mathrm{~mm}]$ \\
\hline With preheating & $0.6[\mathrm{~mm}]$ \\
\hline
\end{tabular}

\section{Prevention of Fountain Flow inside Mold}

Flow Analysis. When the pressing velocity of the upper mold is higher, upward flow causes the fountain inside mold. Hence, we examine pressing condition without fountain. We examine the pressing condition without fountain inside mold by using CFD analysis. The flow analysis targets at upward flowing in a narrow cylindrical path of diameter: $0.010 \mathrm{~m}$. Here the 3D-mesh size is $2 \mathrm{~mm}$ for one block. Liquid inflow velocity from the bottom has been examined instead of changing the pressing velocity just like practical case. From these simulations, the upper limit speed for flowing without fountain is confirmed. The target metal is cast iron. The boundary condition between wall and liquid is set to no-slip.

Here, five observation points are set in cavity as shown in Fig. 5 (b). The decision method of the fountain flow observes the passing order of sampling points at intervals of $1 \mathrm{~mm}$. If the flow keeps steady, liquid flows in the sequence from A to E. On the other hand, fountain flow makes another 


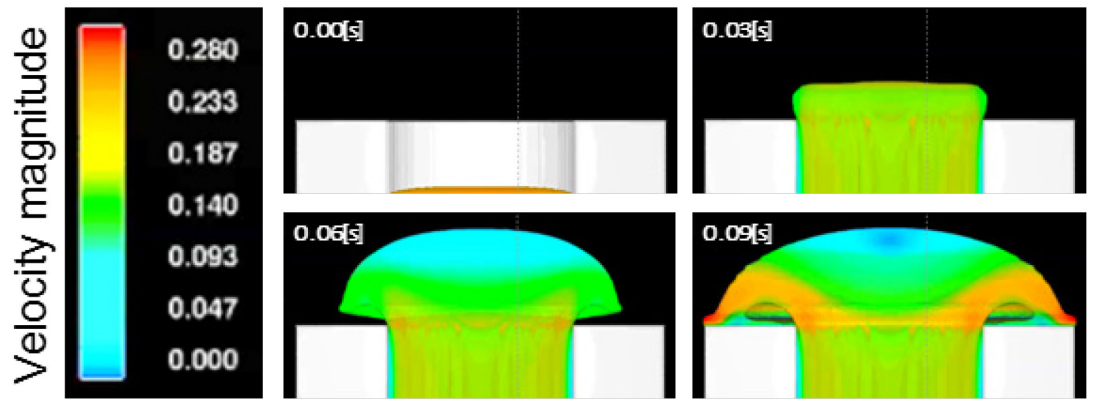

(a) Liquid flow in a series of motion
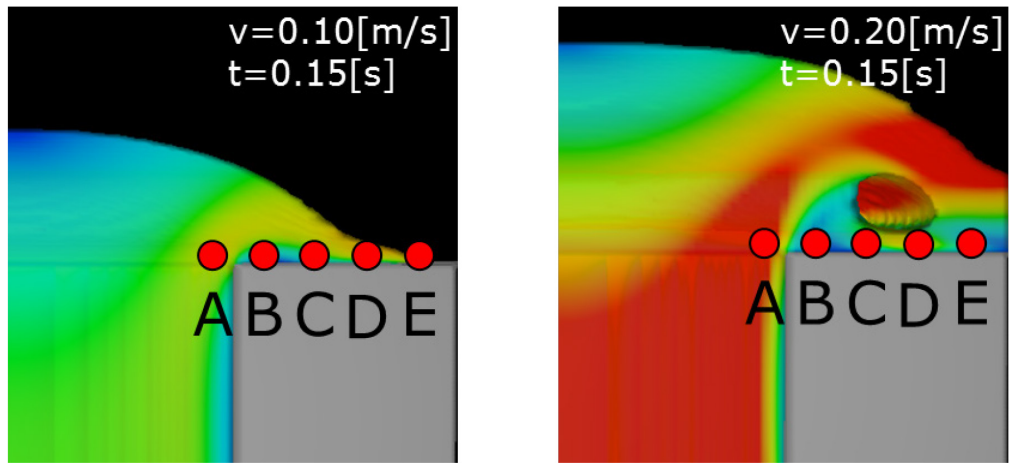

(b) Magnified view at path corner
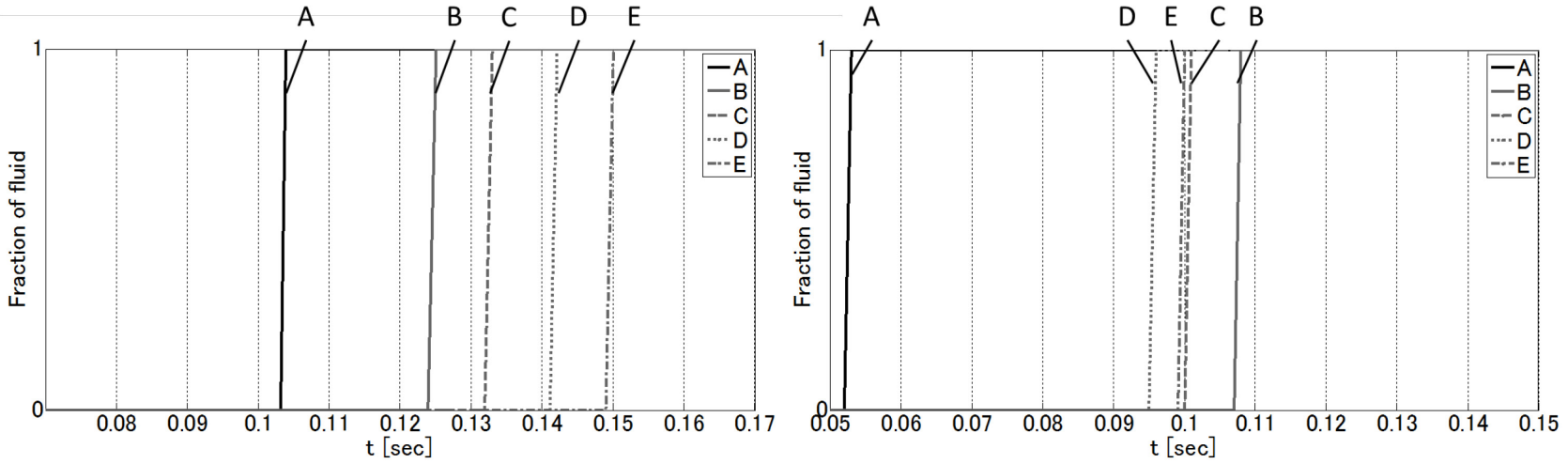

(c) Judgement of fountain

Fig. 5: Liquid flow analysis

sequence. In the case of molten metal passing in order from left as indicated in the Fig. 5 (c), the liquid flow inside mold is steady, otherwise, generates the fountain. From the result of the liquid flow analysis, the constraint speed for steady flow without fountain is given as lower $0.12 \mathrm{~m} / \mathrm{s}$.

Constraint of Pressing Velocity Design. We derived the constraint condition of pressing velocity without fountain based on the previous flow analysis results. The upper limit of upward flow speed $v_{\text {lim }}=0.12 \mathrm{~m} / \mathrm{s}$ in the cavity is constant for the all flow pass shapes. The upper limit of pressing velocity $\dot{z}_{\text {lim }}$ by Eq. 1 using upper mold shape can be derived.

$$
\dot{z}_{\text {lim }}(h)=\frac{A_{\text {fmin }}(h)}{A_{m}(h)} v_{\text {lim }}
$$

$A_{m}$ is the cross-sectional area of upper mold at the hieght of liquid surface in the cavity. $A_{f m i n}$ is the area of the narrowest pass within filled molten metal. In the case of consideration for only the upward speed at the surface (Fig. 6 dash line), flow speed is higher the liquid surface, as a result, upward flow causes the fountain. Therefore, as is the case Fig. 6 (solid line), the upper limit speed don't be raised, no matter how flow pass is wider. In the case of the constraint of pressing velocity as red line, pressing time is $0.65 \mathrm{~s}$ longer than the case of dash line. 


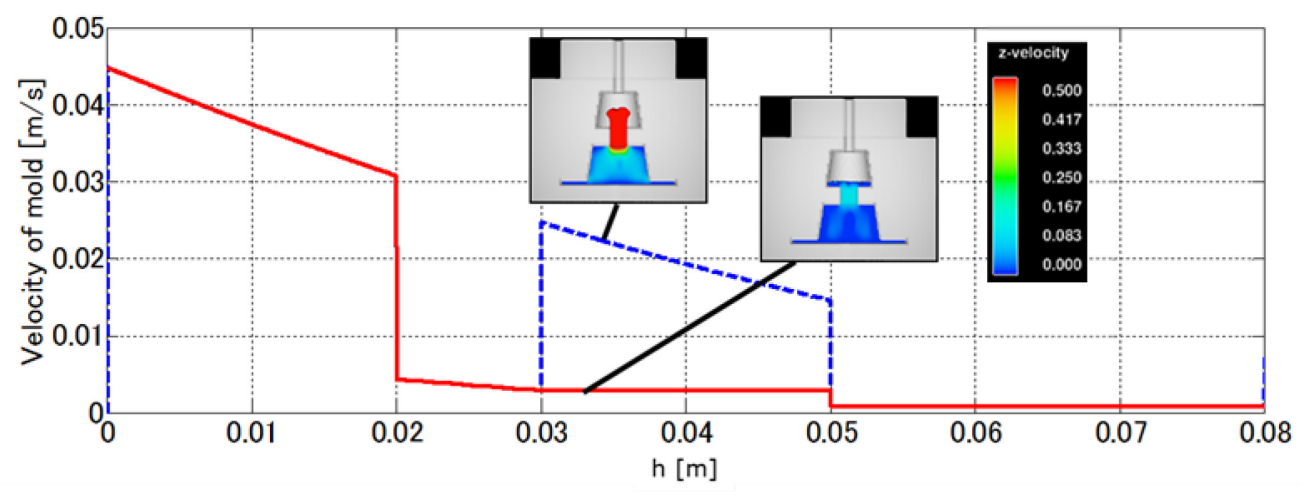

Fig. 6: Pressing velocity design with constraint

\section{Design of Pressing Control Velocity}

In this chapter, we design the control pressing velocity using by the MPC method, based on mentioned above. In this paper, the pressing velocity is designed by the MPC simulation in offline. The designed velocity can be derived by constraint considered the upper limit speed for flowing without fountain and filling pressure.

Model Predictive Control. Pressure control must be satisfied under the pressure constraint condition for high-quality sand mold casting. The MPC method is an useful control technique that predicts a future output of pressure. Eq. 2 is the response of the filling system related to the pressing motion.

$$
\boldsymbol{\tau}=\boldsymbol{y}_{f}+\boldsymbol{S u}
$$

$\boldsymbol{y}_{\boldsymbol{f}}$ is the free response in the future, $\boldsymbol{S}$ is the unit step response matrix, $u$ is the control input. Then, the evaluation function $V(k)$ is defined as follows.

$$
V(k)=\sum_{i=H w}^{H_{p}}\|\boldsymbol{\tau}-\boldsymbol{r}\|_{\boldsymbol{Q}}^{2}=\sum_{i=H \boldsymbol{w}}^{\boldsymbol{H}_{\boldsymbol{p}}}\left\|\boldsymbol{y}_{\boldsymbol{f}}+\boldsymbol{S} u-\boldsymbol{r}\right\|_{\boldsymbol{Q}}^{2}=\sum_{\boldsymbol{i}=\boldsymbol{H} \boldsymbol{w}}^{\boldsymbol{H}_{\boldsymbol{p}}}\|\boldsymbol{Y}+\boldsymbol{S} u\|_{\boldsymbol{Q}}^{2}
$$

The evaluation function $V(k)$ is the deviation of the output response $\boldsymbol{\tau}$ from the reference pressure trajectory $\boldsymbol{r} . \boldsymbol{Q}$ is the weight matrix, details is described below, and $\boldsymbol{Y}=\boldsymbol{y}_{\boldsymbol{f}}-\boldsymbol{r}$. The control input $u$ can be derived, as the evaluation function $V(k)$ is minimum.

$$
\begin{aligned}
& \frac{d V(k)}{d u}=\boldsymbol{S}^{\mathrm{T}} \boldsymbol{Q} \boldsymbol{Y}+\boldsymbol{S}^{\mathrm{T}} \boldsymbol{Q}^{\mathrm{T}} \boldsymbol{Y}+2 \boldsymbol{S}^{\mathrm{T}} \boldsymbol{Q S} u=0 \\
& u=\left(-\boldsymbol{S}^{\mathrm{T}} \boldsymbol{Q} \boldsymbol{S}\right)^{-1}\left(\boldsymbol{S}^{\mathrm{T}} \boldsymbol{Q} \boldsymbol{Y}\right)
\end{aligned}
$$

Next, the weight matrix $\boldsymbol{Q}$ is discussed. It is important that pressing position control in order to end the pressing motion at objective position for high-quality sand mold casting. However, the press casting machine only has an axis, can only control at same time, the pressing position control or the filling pressure control. Therefore, defined the weight matrix $\boldsymbol{Q}$, it can control the pressing position and filling pressure control, changing the weight variable dependent on the output response pressure.

$$
\begin{aligned}
& \boldsymbol{Q}=\left[\begin{array}{cc}
q_{m} \boldsymbol{I} & \mathbf{0} \\
\mathbf{0} & q_{p} \boldsymbol{I}
\end{array}\right] \\
& \begin{cases}q_{\text {rate }}=1 & \left(P>P_{\text {swHigh }}\right) \\
q_{\text {rate }}=0 & \left(P<P_{\text {swLow }}\right) \\
q_{\text {rate }}=\frac{P-P_{\text {swLow }}}{P_{\text {swHigh }}-P_{\text {swLow }}} & (\text { otherwise })\end{cases}
\end{aligned}
$$




$$
\begin{aligned}
& q_{p}=q_{\text {rate }} \\
& q_{m}=1-q_{\text {rate }}
\end{aligned}
$$

As the response pressure $P$ approaches $P_{\text {swHigh }}, q_{p}$ is higher, the control input is derived so that the pressure constraint may not be exceeded. Contrarily, as the response pressure $P$ approaches $P_{\text {swLow }}, q_{m}$ is higher, the control input is derived so that the upper mold moves to the objective position.

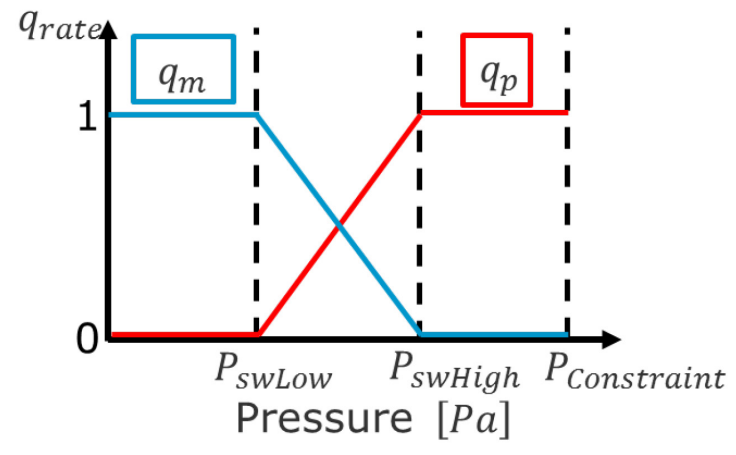

Fig. 7: Weight function of $q_{\text {rate }}$

Control Input Design and Simulation. We designed the controlled pressing velocity, by the MPC simulation in offline. The designed velocity can be derived by constraint considered the upper limit speed for flowing without fountain and pressing machine specification. The simulation result indicates that the MPC design of pressing motion is effective for filling behaviors of flow and pressure in the sand mold press casting method. The designed pressing motion, the metal pressure behavior and the steady flow without fountain were confirmed by CFD.
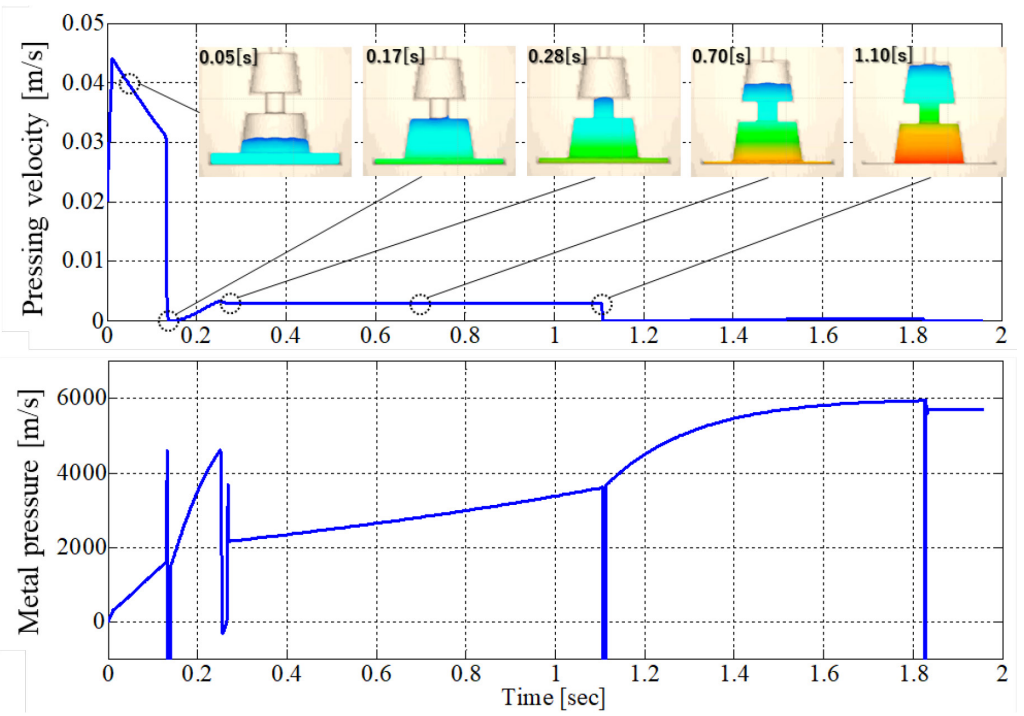

Fig. 8: Model predictive control simulation 


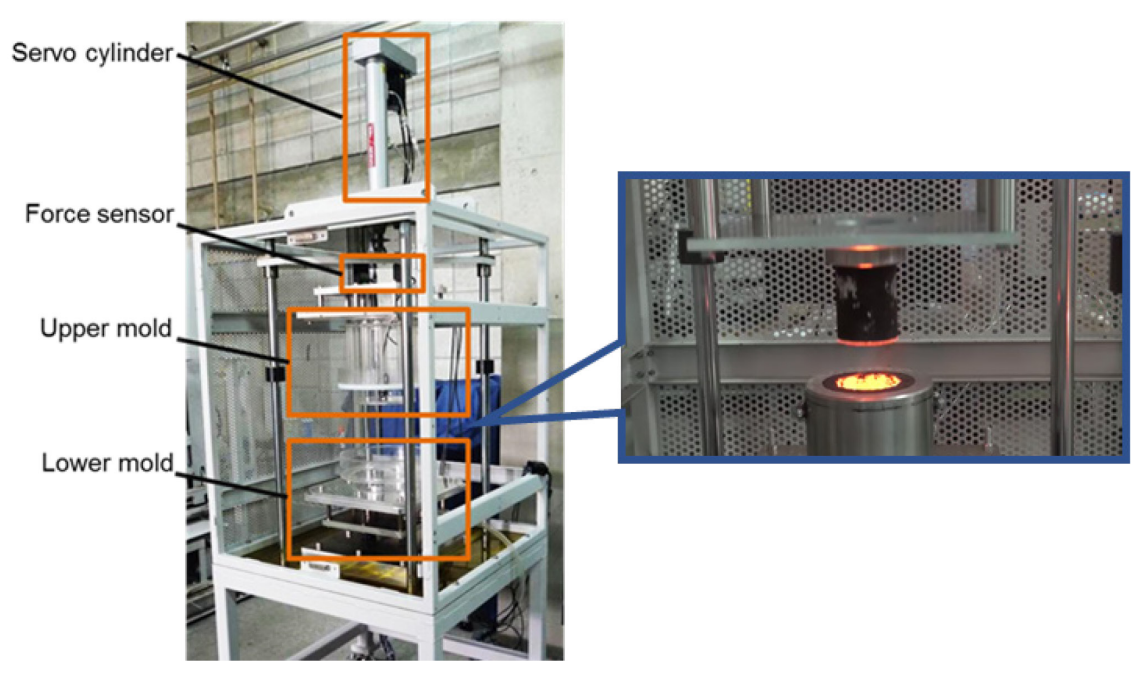

(a) Press casting machine

Fig. 9: Experiment using proposed filling control

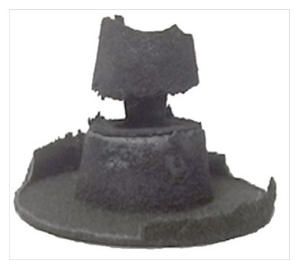

(b) Filling control without flow velocity constraint

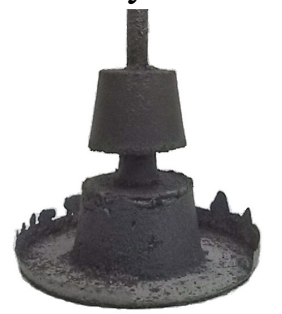

(c) Filling control with constraints

\section{Experimental Result of Sand Mold Press Casting}

The iron casting experiment with ideal filling rate control has been carried out by using the designed pressing velocity as mentioned in Fig. 8. This pressing motion can be accurately operated by feedforward control input to the servo-drive cylinder in Fig. 9 (a). The material is molten metal and the initial temperature of the metal is $1400{ }^{\circ} \mathrm{C}$. The cast products in the experiment are shown in Fig. 9 (b) and (c) have thin burr for both. The molten meta completely filled the cavity and makes target shape in the case of Filling control with constraints for both flow and pressure. The product is without physical penetration on the surface, and expected shape, compared with Fig. 9 (b). We confirmed the designed control pressing motion is successfully performed for actual molten metal experiment in the press casting process.

\section{Conclusion}

We proposed the filling pressure control using MPC and preheating efficiency for solidification prevention. The designed pressing motion, the metal pressure behavior and the steady flow without fountain were confirmed by CFD and molten metal experiment. It is made clear that the mold must be preheat over $200{ }^{\circ} \mathrm{C}$, the press motion and the metal material condition is effective for preventing the solidification of metal before the end of pressing. And the constraint speed for steady flow without fountain is given as lower $0.12 \mathrm{~m} / \mathrm{s}$. We confirmed the designed control pressing motion is effective by experiment. As the near future work, the proposed filling pressure feedback system in real-time will be implemented in the sand mold press casting process. Then, controlled pressing experiments with more complex shape mold will be carried out for evaluating the effectiveness for practical casting production.

\section{References}

[1] J. Campbell, Counter Gravity Casting, In: Nastac L. et al. (eds) Advances in the Science and Engineering of Casting Solidification. Springer, Cham, 2015, pp. 357-362

[2] W. H. Zhu, J. C. Piedboeuf, Adaptive Output Force Tracking Control of Hydraulic Cylinders With Applications to Robot Manipulators, J. Dyn. Syst.-T. ASME 127(2) (2005) 206-217

[3] J. M. Lee, S. H. Park, J. S. Kim, A robust control of the pressure in a control-cylinder for the variable displacement axial piston pump, Proceedings of 7 th Asian Control Conference, pp.1280-1285, 2009 
[4] R. Tasaki, Y. Noda, K. Terashima, K. Hashimoto, Sequence Control of Pressing Velocity for Pressure in Press Casting Process Using Greensand Mold, Int. J. Cast Metal Res. 21 (1-4) (2008) 269-274

[5] R. Tasaki, Y. Noda, K. Hashimoto, K. Terashima, Modeling and Control of Pressurized Molten Metal in Press Casting, Journal of Mechanics Engineering and Automation, 2 (2) (2012) 77-84.

[6] T. Takako, R. Tasaki, K. Hashimoto, K. Terashima, Optimum Feedforward and Model Predictive Control of Molten Metal Pressure in Greensand Mold Press Casting, 72nd World Foundry Congress, No.O-152, 2016 